\title{
A GROUP WITH DEEP POCKETS FOR ALL FINITE GENERATING SETS
}

\author{
ANDREW D. WARSHALL
}

\begin{abstract}
We show that the discrete Heisenberg group has unbounded deadend depth with respect to every finite generating set. We also show that, in contrast, it has bounded retreat depth.
\end{abstract}

\section{INTRODUCTION}

Let $G$ be any group and let $A$ be a generating set for $G$. Let $\|g\|_{A}$ denote the length of the minimal-length word in $A$ representing $g$. By the depth (or dead-end depth) of an element $g \in G$ with respect to $A$ we mean the distance (in the word metric with respect to $A$ ) from $g$ to the nearest $g^{\prime} \in G$ with $\left\|g^{\prime}\right\|_{A}>\|g\|_{A}$. If there is no such $g^{\prime}$, then the depth of $g$ is infinite.

If $G$ is a finite group then it will have elements of infinite depth. In contrast, this cannot happen for $G$ infinite, provided $A$ is finite. It is natural then to ask whether depth is bounded over all group elements. The first result in this direction was due to Bogopol'skii, who showed in [2] that every infinite hyperbolic group has a bound on the depth of its elements with respect to any given finite generating set. Later, we showed the same in 7 for all infinite Euclidean groups, and Lehnert then showed it in [5] for all groups with more than one end.

It is not difficult, however, to construct infinite finitely generated groups $G$ and generating sets $A$ such that there is no bound on the depth of elements of $G$ with respect to $A$. The first example, given by Cleary and Taback in 4, was the lamplighter group, namely $\mathbb{Z}_{2} \prec \mathbb{Z}=\left\langle a, t \mid t^{2},\left[a, a^{t^{i}}\right], i \in \mathbb{N}\right\rangle$, with respect to the generating set $\{a, t\}$. Although this group is not finitely presented, this is not essential; a (more complicated) finitely presented example was given by Cleary and Riley in [3].

However, the property of having unbounded depth (also known as the deep pockets property) is not a generating-set invariant; this was shown in joint work between this author and Riley in [6]2 Thus the question remains of whether there exists an infinte group which has unbounded depth with respect to every finite generating set. In this paper, we answer this question in the affirmative.

Date: October 24, 2018.

2000 Mathematics Subject Classification. 20F65.

We thank our advisor, Andrew Casson, for his helpful comments.

${ }^{1}$ The published version of this paper contained an error, pointed out by Lehnert. A corrected version is available from the arXiv.

${ }^{2}$ The published version of this paper contained an analogous error, which became clear after the error in [3] was pointed out; however, the proof of Theorem 3 contained in Sections 2 and 3 is unaffected. 
In particular, let $H$ denote the discrete Heisenberg group

$$
\langle x, y \mid[x,[x, y]],[y,[x, y]]\rangle \text {. }
$$

Then we will prove the following

Proposition 1. Let $A$ be a finite generating set for $H$. Then there is no bound on the depth of all elements of $H$ with respect to $A$.

Definition 1. Let $G$ be a group, $A$ a generating set for $G$. Let $g \in G$ be such that $\|g\|_{A}=l$. Then the retreat depth of $g$ with respect to $A$ is the minimal $d$ such that $g$ lies in an unbounded component of $B_{l-d}(1)$. (Lehnert in [5] refers to a concept similar to this as strong depth.)

The above definition is motivated by Bowditch's Question 8.4 in [1, which asks in effect whether, for every infinite $G$ and finite $A$, retreat depth is bounded for all $g \in G$. (This question was answered in the negative by Erschler, using the same example of the lamplighter group.) It is clear that if (with respect to some fixed generating set) the depth of elements of a group is bounded, then so is the retreat depth. The converse, however, fails fairly resoundingly for the Heisenberg group.

Proposition 2. Let $A$ be a finite generating set for $H$. Then there exists $r \in \mathbb{N}$ such that, for every $h \in H$, the retreat depth for $h$ is $\leq r$.

The paper is organized is follows. In the brief Section 2, we will remind the reader of some facts about $\mathbb{Z}^{2}$ and metric geometry, the proofs of which can be found in [7, and fix some notations. In Section 3, we will give the proof of Proposition 1 , Finally, in Section 4, we will give the proof of Proposition 2,

\section{Preliminaries}

To prove either of the main results of this paper, we will first need to recall some facts about the geometry of $\mathbb{Z}^{2}$ with respect to arbitrary finite generating sets. Let $A$ be such a generating set and let $B$ be the (closed) convex hull of $A \cup A^{-1}$. Then $B$ is a polygon in $\mathbb{R}^{2}$ and we have the following

Proposition 3. Let $a_{1}, a_{2} \in A \cup A^{-1}$ be adjacent vertices of $B$ and let $i_{1}, i_{2} \in \mathbb{Z}_{\geq 0}$. Then $i_{1} a_{1}+i_{2} a_{2}$ is a geodesic word in $A$. In particular, there are $C$ and $D \in \mathbb{N}$ depending only on $A$ such that every $v \in \mathbb{Z}^{2}$ is within $C$ in the standard $L^{1}$ norm and $D$ in the norm with respect to $A$ of some $v^{\prime} \in \mathbb{Z}^{2}$ represented by a geodesic word of this form. It follows that $\|v\|_{A}-D \leq\left\|v^{\prime}\right\|_{A} \leq\|v\|_{A}+D$ and that, if $\|\cdot\|_{B}$ is taken to mean the norm whose unit ball is $B$, then $\left|\|v\|_{A}-\|v\|_{B}\right|$ is bounded independently of $v$.

We omit the proof; for details see [7].

For the first result we will also need an auxiliary fact, having nothing to do with groups.

Proposition 4. Let $f$ be a function from a metric space $A$ to $\mathbb{Z}$ and $n \in \mathbb{Z}$. Suppose there exists $a \in A$ and $r \in \mathbb{Z}$ such that for all $a^{\prime} \in B_{r}(a), f\left(a^{\prime}\right) \leq f(a)+n$. Then there exists some $a^{\prime} \in A$ such that $f$ attains a maximum on $B_{r / n}\left(a^{\prime}\right)$ at $a^{\prime}$.

The proof of this proposition may also be found in [7. Its relevance is that, to prove that the depth of a group is infinite, we may replace distance from the identity with any other function differing from it by at most a finite additive constant.

We agree to use $x$ and $y$ to denote the two standard generators of $H$ and $\phi: H \rightarrow$ $H_{a b}$ to denote the abelianization map. 


\section{Proof of Proposition 1}

We begin by showing the existence of a particularly nice collection of words of "almost minimal" length.

Proposition 5. Let $A$ be a generating set for $H$. Then there exist $G, I \in \mathbb{R}, i_{1}$, $i_{2}, \ldots \in \mathbb{N} \cup\{0\}$ and a sequence of words $w_{00}, w_{10}, \ldots, w_{1 i_{1}}, w_{20}, \ldots, w_{2 i_{2}}, w_{30}$, ... in the letters of $A \cup A^{-1}$ such that

- $l\left(w_{n i}\right)=2 n$ for all $n$ and $i$,

- for all $n, w_{(n+1) 0}$ is obtained from $w_{n i_{n}}$ by inserting a letter of $A$ and its inverse at positions in $w_{n i_{n}}$ separated by one letter,

- for all $n>0$ and $i, w_{n(i+1)}$ is obtained from $w_{n i}$ by interchanging one of the letters inserted to produce $w_{n 1}$ with an adjacent letter,

- each $w_{n i}$ represents $[x, y]^{k_{n i}}$, where for every $m \in \mathbb{N}$ there are $n$ and $i$ such that $\left|m-k_{n i}\right| \leq G$ and

- for all $n$ and $i, l\left(w_{n i}\right)-\left\|[x, y]^{k_{n i}}\right\|_{A} \leq I$.

For $A$ a generating set for $H$ and $n \in \mathbb{Z}_{\geq 0}$, let

$$
I_{A}(n)=\max \left\{|k| \mid\left\|[x, y]^{k}\right\|_{A}=n\right\} .
$$

Proposition 5 will now follow from

Proposition 6. Let $A$ be a generating set for $H$. Then there exist $D, E$ and $F \in \mathbb{R}$ with $E>0$ and for each $n \in \mathbb{N} \cup\{0\}$ a word $w_{n}$ in the letters of $A$ and their inverses such that

- $l\left(w_{n}\right)=2 n$,

- each $w_{n}$ is obtained from $w_{n-1}$ by inserting a letter of $A$ and its inverse (not in general adjacently to each other) and

- each $w_{n}$ represents $[x, y]^{k_{n}}$, where $I_{A}(2 n)-D n \leq k_{n} \leq I_{A}(2 n)$ and $E n^{2}-$ $F n \leq k_{n} \leq E n^{2}$.

We postpone the proof.

Proof of Proposition 5 assuming Proposition [6. We proceed inductively on the assumption that

$$
w_{(n-1) i_{n-1}}=w_{n-1}
$$

from Proposition [6. Let the two letters added to $w_{n-1}$ to make $w_{n}$ be $a$ and $a^{-1}$. Let $w_{n 1}$ be obtained by inserting $a$ and $a^{-1}$ at positions separated by one letter. Then, let $w_{n 2}, \ldots, w_{n i_{n}}$ be obtained by interchanging either $a$ or $a^{-1}$ with an adjacent letter at each step to yield eventually $w_{n i_{n}}=w_{n}$. Note that we can arrange that $i_{n} \leq 2 n$. This construction clearly fulfills the first three conditions.

Since each $w_{n i}$ contains each letter and its inverse an equal number of times it represents some power of $[x, y]$. But each step in the above construction changes the exponent by at most

$$
M_{A}^{\prime}=\max \left\{|k| \mid\left[a_{1}, a_{2}\right]=[x, y]^{k}, a_{1}, a_{2} \in A \cup A^{-1}\right\} .
$$

Since $w_{00}$ represents the identity and $w_{n i_{n}}=w_{n}$ represents $[x, y]^{k_{n}}$ where $k_{n} \geq$ $E n^{2}-F n$ and $E$ and $F$ are independent of $n$ as in Proposition [6, the $k_{n i}$ must increase without bound. Thus the fourth condition is proven if we let $G=M_{A}^{\prime}$.

Set $k_{\text {max }}(A)=\max \left\{|k| \mid x^{i} y^{j}[x, y]^{k} \in A\right\}$. Each $w_{n i}(n>0)$ represents

$$
[x, y]^{k_{n i}}
$$


where

$$
\begin{aligned}
k_{n i} \geq k_{n-1}-2 n M_{A}^{\prime} & \geq I_{A}(2(n-1))-D n-2 n M_{A}^{\prime} \\
& \geq 4(n-C-1)^{2} M_{A}-2(n+C-1) k_{\max }(A)-D n-2 n M_{A}^{\prime} .
\end{aligned}
$$

For $n$ sufficiently large, letting $J=\left(D+2 M_{A}^{\prime}+2 k_{\max }(A)\right) /\left(4 M_{A}\right)+C+2$, this is $\geq 4(n-J)^{2} M_{A}+2(n-J) k_{\max }(A) \geq I_{A}(2 n-2 J)$, where the second inequality holds by the definitions of $I_{A}, k_{\max }(A)$ and $M_{A}$. This proves the final condition, since the $w_{n i}$ are thus within $2 J$ of minimal length. (The finitely many insufficiently large values of $n$ can only increase $I$ by a finite amount.)

Proposition 7. Let $A$ be a generating set for $H$. Then there exist $C$ (= $G$ from Proposition 5), $D$ and $E \in \mathbb{Z}$ with the following property. For every $n \in \mathbb{Z}$ there exists $n^{\prime} \in \mathbb{Z}$ with $\left|n-n^{\prime}\right| \leq C$ such that, if $v=v_{1} \phi(x)+v_{2} \phi(y) \in H_{a b}$ with $\|v\|_{\{\phi(x), \phi(y)\}} \leq \sqrt[6]{|n|}$, then there exist words $w$ and $w^{\prime}$ in the letters of $A$ and their inverses such that

- $w$ represents $[x, y]^{n^{\prime}}$

- $l(w) \leq\left\|[x, y]^{n^{\prime}}\right\|_{A}+D$,

- $w$ can be obtained from some word in the sequence given by Proposition 5 by cyclic permutation and possibly inverting all letters,

- $\phi\left(w^{\prime}\right)=v$,

- $l(w) \geq l\left(w^{\prime}\right)-E$ and

- $l\left(w^{\prime} w^{-1}\right) \leq \sqrt[5]{\left|n^{\prime}\right|}$

Remark. Let $w^{\prime}$ represent $x^{v_{1}} y^{v_{2}}[x, y]^{n^{\prime \prime}}$. Then, since $\left\|x^{v_{1}} y^{v_{2}}[x, y]^{n^{\prime \prime}-n^{\prime}}\right\|_{A} \leq \sqrt[5]{\left|n^{\prime}\right|}$, it follows that there is some $F$ depending only on $A$ such that $\left|n^{\prime \prime}-n\right| \leq F\left|n^{\prime}\right|^{2 / 5}+$ C.

We will need the following proposition, which asserts basically that minimallength representatives of powers of $[x, y]$ cannot be too long and thin.

Proposition 8. Let $A$ be a generating set of $H$. Then there are $C, D$ and $E \in \mathbb{R}$ such that the following is true. Let $a_{1} \ldots a_{m}$ be a minimal-length word in the letters of $A$ such that $a_{1} \ldots a_{m}=[x, y]^{n}$. Let $f: H_{a b} \rightarrow \mathbb{R}$ be linear of norm 1 . Then there is a subword $a_{i} \ldots a_{j}$ of $a_{1} \ldots a_{m}$ with $C \sqrt{|n|} \geq j-i \geq D|\sqrt{n}|-E$ and $C \sqrt{|n|} \geq\left|f\left(\phi\left(a_{i} \ldots a_{j}\right)\right)\right| \geq D \sqrt{|n|}-E$.

Lemma 9. Let $A$ be a generating set of $H$. Then there are $C, D$ and $E \in \mathbb{R}$ such that the following is true. Let $a_{1} \ldots a_{m}$ be a minimal-length word in the letters of $A$ such that $a_{1} \ldots a_{m}=[x, y]^{n}$. Let $f: H_{a b} \rightarrow \mathbb{R}$ be linear of norm 1 . Let $P_{n}$ be $\left\{e, a_{1}, a_{1} a_{2}, \ldots, a_{1} \ldots a_{m}=[x, y]^{n}\right\}$. Then the diameter of $f\left(\phi\left(P_{n}\right)\right)$ is at most $C \sqrt{|n|}$ and at least $D \sqrt{|n|}-E$.

Proof. Let $M(A)=\max _{a \in A}\|a\|_{\{x, y\}}$. We know that there is $F \in \mathbb{R}$ such that $m \leq F \sqrt{|n|}$, since this is so with respect to the standard generating set. Thus we know that the diameter of $P_{n}$ with respect to the standard generators of $H$ is at most

$$
m M(A) \leq F M(A) \sqrt{|n|} .
$$


Since neither $f$ nor $\phi$ increases distances (where we consider $H_{a b}$ with respect to the images of the standard generators) the same bound holds for the diameter of $f\left(\phi\left(P_{n}\right)\right)$, so we may take $C=F M(A)$.

Conversely, choose a minimal-length word in the standard generators for each letter of $A$, so $a_{1} \ldots a_{m}$ becomes $b_{1} \ldots b_{M}, b_{i} \in\left\{x, y, x^{-1} \cdot y^{-1}\right\}, M \leq M(A) m$. Then, if

$$
g: H_{a b} \rightarrow \mathbb{R}=f \circ\left(\begin{array}{cc}
0 & 1 \\
-1 & 0
\end{array}\right),
$$

(where the matrix is with respect to the basis $\langle\phi(x), \phi(y)\rangle$ ) we have

$$
\begin{gathered}
|n|=\left|\sum_{i=1}^{M} g\left(b_{i}\right)\left[f\left(\phi\left(b_{1} \ldots b_{i-1}\right)\right)+\frac{f\left(\phi\left(b_{1}\right)\right)}{2}\right]\right| \\
\leq \sum_{i=1}^{M}\left|g\left(b_{i}\right)\right| \cdot\left[\max _{i \in\{1, \ldots, M\}}\left|f\left(\phi\left(b_{1} \ldots b_{i-1}\right)\right)\right|+\frac{\max _{b \in\{x, y\}}|f(\phi(b))|}{2}\right. \\
\leq M\left[\max _{i \in\{0, \ldots, m\}}\left|f\left(\phi\left(a_{1} \ldots a_{i}\right)\right)\right|+M(A)+\frac{1}{2}\right] \\
\leq M(A) m\left[\max _{i \in\{0, \ldots, m\}}\left|f\left(\phi\left(a_{1} \ldots a_{i}\right)\right)\right|+2 M(A)\right. \\
\leq F M(A) \sqrt{|n|}\left[\max _{i \in\{0, \ldots, m\}}\left|f\left(\phi\left(a_{1} \ldots a_{i}\right)\right)\right|+2 M(A) .\right.
\end{gathered}
$$

But the diameter of $f\left(\phi\left(P_{n}\right)\right)$ is $\geq \max _{i \in\{0, \ldots, m\}}\left|f\left(\phi\left(a_{1} \ldots a_{i}\right)\right)\right|$, so by the above it is

$$
\geq \frac{\sqrt{|n|}}{F M(A)}-2 M(A)
$$

Thus we may take $D=1 /(F M(A))$ and $E=2 M(A)$.

Proof of Proposition 8. Lemma 9 gives us $C, D$ and $E$ independent of $n$ and some subword $a_{i} \ldots a_{j}$ such that $C \sqrt{|n|} \geq\left|f\left(\phi\left(a_{i} \ldots a_{j}\right)\right)\right| \geq D \sqrt{|n|}-E$. But since $f$ and $\phi$ do not increase distances, we know $j-i \geq D \sqrt{|n|}-E-1$. Finally, $j-i<m \leq F \sqrt{|n|}$ for some $F$ depending only on $A$. Since we can absorb the 1 into $E$ and redefine $C$ to be the greater of $C$ and $F$, we are done.

Proof of Proposition 7. Let $C=G$ and $D=I$ from Proposition 5 , and let $w$ representing $[x, y]^{n^{\prime}}$ be the word given by that proposition with $\left|n-n^{\prime}\right| \leq C$. Then the first three conditions hold.

Let $v \in H_{a b}$ be such that $\|v\|_{\{\phi(x), \phi(y)\}} \leq \sqrt[6]{\left|n^{\prime}\right|}$ but no $w^{\prime}$ exists satisfying the remaining conditions. As in the discussion before Proposition 3 , identifying $H_{a b}$ with $\mathbb{Z}^{2}$, let $B$ be the closed convex hull of $\pm \phi(A)$ and let $\|\cdot\|_{B}$ be the norm with $B$ as its unit ball. Then Proposition 3 gives $F$ such that, for all $v^{\prime} \in H_{a b}$, $\left|\left\|v^{\prime}\right\|_{B}-\left\|v^{\prime}\right\|_{\phi(A)}\right| \leq F$. Express $w=w_{1} w_{2}$, where $l\left(w_{1}\right)=\sqrt[5]{n^{\prime}} / 2-2 F$. It follows (setting $E=2 F$ ) that $\left\|\phi\left(w_{1}\right)+v\right\|_{B}=\left\|\phi\left(w_{2}\right)-v\right\|_{B}>\sqrt[5]{n^{\prime}} / 2-F$. However, everything we know about $w$ applies equally to any cyclic permutation of $w$ and to any word obtained by inverting every letter of $w$. We conclude that, for any cyclic subword $u$ of $w$ with $l(u)=\sqrt[5]{n^{\prime}} / 2-2 F,\|\phi(u) \pm v\|_{B}>\sqrt[5]{n^{\prime}} / 2-F$.

However, $\|\phi(u)\|_{B} \leq l(u)+F=\sqrt[5]{n^{\prime}} / 2-F$ for any such subword $u$. Let $B^{\prime}$ be the dilation of $B$ by $\sqrt[5]{n^{\prime}} / 2-F$. Then $\phi(u) \in B^{\prime}$ while $\phi(u) \pm v \notin B^{\prime}$. We refer to points on the boundary of $B^{\prime}$ where the lower (respectively upper) directional 
derivative with respect to $v$ of $\|\cdot\|_{B}$ is positive (resp. negative) as $v$-positive (resp. $v$-negative). Since, for any point in the interior of an edge, this is equivalent to the outward normal to the edge's having positive (resp. negative) dot product with $v$, we can speak of edges' being $v$-positive or $v$-negative if we restrict attention to their interior. Furthermore, a vertex of $B$ is $v$-positive (respectively $v$-negative) iff both edges incident to it are. In this language, $\phi(u)$ lies within $\|v\|$ (in the Euclidean norm) of both a point on a $v$-positive edge of $B^{\prime}$ and one on a $v$-negative edge.

Since $B^{\prime}$ is convex and simple, there is $G$ such that $\phi(u)$ is within $G\|v\|$ of a vertex of $B^{\prime}$ at which a $v$-positive and a $v$-negative edge meet. If there are any such vertices (as there must bem by assumption), there are exactly two, and they lie opposite to each other with respect to the origin. Call them $\pm c$ and let $V \subset \mathbb{Z}^{2}$ be the subspace spanned by $c$. Let $f: \mathbb{Z}^{2} \rightarrow V^{\perp}$ be orthogonal projection, so that ker $f=V$. But then Proposition 8 gives $I$ depending only on $A$ such that for $n$ large enough there is a cyclic subword $u$ of $w$ of length $\sqrt[5]{n^{\prime}} / 2-2 F$ such that $\|f(\phi(u))\|>I \sqrt[5]{n^{\prime}}$. (If $n$ is not large enough, we just set $n^{\prime}=0$, increasing $C$ as needed.) But if we let $t_{\max }(A)=\max _{a \in A}\|\phi(A)\|$ then $\|v\| \leq t_{\max } \sqrt[6]{n^{\prime}}$, so $\|f(\phi(u))\| \leq G\|v\|+\|f(c)\|=G\|v\| \leq G t_{\max }(A) \sqrt[6]{n^{\prime}}$, which is a contradiction for $n>\left(G t_{\max }(A) / I\right)^{30}+C$, so we are done if we let $E=2 F$.

Proof of Proposition 1, Let $n \in \mathbb{N}$ and let $n^{\prime}$ be as in Proposition 7, Let $x^{i} y^{j}[x, y]^{k}$ be such that $\left\|x^{i} y^{j}[x, y]^{k-n}\right\|_{A} \leq \sqrt[6]{n^{\prime}}$. Let $v=(i, j)$ and let $w, w^{\prime}, n^{\prime \prime}, C, D$ and $E$ the words and constants given by that proposition. Then $l(w) \leq\left\|[x, y]^{n^{\prime}}\right\|_{A}+D \leq$ $\left\|[x, y]^{n}\right\|_{A}+C\|[x, y]\|_{A}+D$.

By the remark following the statement of Proposition 7 , there is some $F$ depending only on $A$ such that

$$
\left|n^{\prime \prime}-n\right| \leq F\left|n^{\prime}\right|^{2 / 5}+C
$$

Since $\left\|x^{i} y^{j}[x, y]^{k-n}\right\|_{A} \leq \sqrt[6]{n^{\prime}}$, it follows that $\left\|x^{i} y^{j}[x, y]^{k-n}\right\|_{\{x, y\}} \leq 2 \sqrt{G} \sqrt[6]{n^{\prime}}$ for sone $G>0$ depending only on $A$, whence $|k-n| \leq G \sqrt[3]{n^{\prime}}$. Thus

$$
\left|n^{\prime \prime}-k\right| \leq\left|n^{\prime \prime}-n\right|+|n-k| \leq F\left|n^{\prime}\right|^{2 / 5}+G \sqrt[3]{n^{\prime}}+C \leq(F+G)\left|n^{\prime}\right|^{2 / 5}+C .
$$

Applying Proposition 5 to $k+n^{\prime}-n^{\prime \prime}$ gives some $w^{\prime \prime}$ representing $[x, y]^{n^{\prime \prime \prime}}$ with $\left|n^{\prime \prime \prime}+n^{\prime \prime}-n^{\prime}-k\right| \leq C$, so

$$
\begin{aligned}
\left|n^{\prime \prime \prime}-n\right| \leq \mid n^{\prime \prime \prime}+n^{\prime \prime}- & n^{\prime}-k|+| n^{\prime \prime}-k|+| n^{\prime}-n \mid \\
& \leq C+(F+G)\left|n^{\prime}\right|^{2 / 5}+C+C=(F+G)\left|n^{\prime}\right|^{2 / 5}+3 C .
\end{aligned}
$$

It follows from Propositions 5 and 7 that a cyclic permutation, combined with possibly inverting every element, transforms $w^{\prime \prime}$ into a word that differs from $w$ only in changing the position of boundedly many letters (say by $I$ ) and adding or deleting at most $I$ letters. Assume without loss of generality that $w^{\prime \prime}$ is so transformed. Decompose $w=w_{1} w_{2}$ and $w^{\prime}$ as $w_{1}^{\prime} w_{2}$, so that $l\left(w_{1}\right) \leq \sqrt[5]{n^{\prime}}$ and $l\left(w_{1}^{\prime}\right) \leq l\left(w_{1}\right)+E$. Note that $w_{1}^{\prime} w_{1}^{-1}=w^{\prime} w^{-1}=x^{i} y^{j}[x, y]^{n^{\prime \prime}-n^{\prime}}$. Finally, decompose $w^{\prime \prime}=w_{1}^{\prime \prime} w_{2}^{\prime \prime}$, where $l\left(w_{1}^{\prime \prime}\right)=l\left(w_{1}\right)$. (We may safely neglect the case where $l\left(w^{\prime \prime}\right)<l\left(w_{1}\right)$, for then $n^{\prime}-\sqrt[5]{n^{\prime}}<I$, yielding a bounded number of cases which may be ignored.) Let $t_{\text {max }}(A)$ again denote $\max _{a \in A} \phi(a)$ and let $t_{\text {max }}^{\prime}(A)$ denote $\max \left(\|x\|_{A},\|y\|_{A}\right)$. Then $\left\|\phi\left(w_{1}^{\prime \prime}\right)-\phi\left(w_{1}\right)\right\| \leq I t_{\max }(A)$. Let

$$
M_{A}^{\prime}=\max \left\{|k| \mid\left[a_{1}, a_{2}\right]=[x, y]^{k}, a_{1}, a_{2} \in A \cup A^{-1}\right\} .
$$


Then there is a word $u^{\prime}$ with $l\left(u^{\prime}\right) \leq I t_{\max }(A) t_{\max }^{\prime}(A)$ with $\phi\left(u^{\prime}\right)=\phi\left(w_{1}^{\prime \prime}\right)-\phi\left(w_{1}\right)$ and $w_{1}^{-1} w_{1}^{\prime \prime}=u^{\prime}[x, y]^{k}$, where $k \leq l\left(u^{\prime}\right) l\left(w_{1}\right) M_{A}^{\prime}$. This yields a word $u$ representing $w_{1}^{-1} w_{1}^{\prime \prime}$ with

$$
\begin{aligned}
& l(u) \leq I t_{\max }(A) t_{\text {max }}^{\prime}(A)+8 \sqrt{G k} \\
& \leq I t_{\max }(A) t_{\text {m }}^{\prime} a x(A)+8 \sqrt{G l\left(u^{\prime}\right) l\left(w_{1}\right) M_{A}^{\prime}} \\
& \quad \leq I t_{\max }(A) t_{\max }^{\prime}(A)+8 \sqrt{G I t_{\max }(A) t_{\max }^{\prime}(A) M_{A}^{\prime}} \sqrt[10]{n^{\prime}} .
\end{aligned}
$$

Thus $w_{1}^{\prime} u w_{2}^{\prime \prime}$ has length (for some $J$ depending only on $A$ )

$$
\begin{aligned}
\leq l\left(w_{1}^{\prime}\right) & +l(u)+l\left(w_{2}^{\prime \prime}\right) \\
\leq l\left(w_{1}\right)+ & E+I t_{\max }(A) t_{\max }^{\prime}(A)+8 \sqrt{G I t_{\max }(A) t_{\max }^{\prime}(A) M_{A}^{\prime}} \sqrt[10]{n^{\prime}}+l\left(w_{2}\right)+I \\
& =l(w)+8 \sqrt{G I t_{\max }(A) t_{\max }^{\prime}(A) M_{A}^{\prime}} \sqrt[10]{n^{\prime}}+I t_{\max }(A) t_{\max }^{\prime}(A)+E+I
\end{aligned}
$$

and represents $w_{1}^{\prime} w_{1}^{-1}[x, y]^{n^{\prime \prime \prime}}=x^{i} y^{j}[x, y]^{n^{\prime \prime \prime}+n^{\prime \prime}-n^{\prime}}$. Since

$$
\left\|n^{\prime \prime \prime}+n^{\prime \prime}-n^{\prime}-k\right\| \leq C,
$$

there is a word of length at most

$$
l(w)+8 \sqrt{\operatorname{GIt}_{\max }(A) t_{\max }^{\prime}(A) M_{A}^{\prime}} \sqrt[10]{n^{\prime}}+I t_{\max }(A) t_{\max }^{\prime}(A)+E+I+C\|[x, y]\|_{A}
$$

representing $x^{i} y^{j}[x, y]^{k}$. Since

$$
l(w) \leq\left\|[x, y]^{n^{\prime}}\right\|_{A}+D \leq\left\|[x, y]^{n}\right\|_{A}+C\|[x, y]\|_{A}+D,
$$

this length bound is $\leq\left\|[x, y]^{n}\right\|_{A}+K \sqrt[10]{n^{\prime}}+L$, where

$$
K=8 \sqrt{G I t_{\text {max }}(A) t_{\text {max }}^{\prime}(A) M_{A}^{\prime}}
$$

and $L=I t_{\max }(A) t_{\text {max }}^{\prime}(A)+E+I+2 C\|[x, y]\|_{A}+D$ both depend only on $A$. Since $\lim _{n \rightarrow \infty} \sqrt[6]{n^{\prime}} /\left(K \sqrt[10]{n^{\prime}}+L\right)=\infty$, we are done by Proposition 4

It remains to prove Proposition 6. We begin with a definition.

Definition 2. Let the isoperimetric constant of a polygon in $\mathbb{R}^{2}$ with respect to a given norm be the ratio of its enclosed area (in the standard measure on $\mathbb{R}^{2}$ ) to the sum of its side lengths with respect to the given norm (which we call the perimeter with respect to that norm).

Lemma 10. Let $\|\cdot\|$ be a norm on $\mathbb{Z}^{2}$ whose fundamental polygon is actually a Euclidean polygon. Then there exists a polygon in $\mathbb{R}^{2}$ with maximal isoperimetric constant among all polygons in $\mathbb{Z}^{2}$ such that

- it is convex and simple,

- each side is parallel to another side of the same length and

- every side is parallel to the ray from the origin to some vertex of the fundamental polygon.

Proof. Any polygon with maximal isoperimetric constant is clearly convex and simple. For any such polygon, circumscribe it with a polygon each side of which is parallel to the ray from the origin to some vertex of the fundamental polygon. This polygon will have at most the same perimeter and at least as large an area. Thus 
there exists a polygon with maximal isoperimetric constant satisfying the first and third conditions.

For the second condition, choose a vertex of the polygon and consider the other point of the polygon which divides the perimeter into two equal parts. A line segment connecting these two points must then divide the area into two equal parts as well, for otherwise the isoperimetric constant would not be maximal. Thus, if we replace one half of the polygon with the other half rotated by $\pi$ and translated, the area, hence the isoperimetric constant, will be the same as before, so still maximal. The result must fulfill the third condition if the original polygon did, and if it fails to be convex and simple then it does not have maximal isoperimetric constant, so neither did the original, a contradiction.

Lemma 11. Let $m \in \mathbb{N}$ and $b_{1}, b_{2}, \ldots, b_{m} \in \mathbb{R}_{+}$such that $\sum_{i=1}^{m} b_{i}=1$. Then for every $n \in \mathbb{N} \cup\{0\}$ and $i \in\{1, \ldots, m\}$ there exists $b_{n i} \in \mathbb{N} \cup\{0\}$ such that

- $b_{0 i}=0$ for all $i$,

- $\sum_{i=1}^{m} b_{n i}=n$ for all $n$,

- $b_{n i}$ is a nondecreasing function of $n$ for all $i$ and

- $\left|b_{n i}-n b_{i}\right|<m$ for all $n$ and $i$.

Remark. It follows that for every $n$ there is some $j \in\{1, \ldots, m\}$ such that $b_{n j}=$ $b_{(n-1) j}+1$ and $b_{n i}=b(n-1) i$ for all $i \neq j$.

Proof. We construct the $b_{n i}$ inductively. First, let all the $b_{0 i}=0$. Assuming the $\left.b_{(} n-1\right) i$ constructed, let $j \in\{1, \ldots, m\}$ be such that $n b_{j}-b_{(n-1) j} \geq n b_{i}-b_{(n-1) i}$ for all $i \in\{1, \ldots, m\}$. Then let $b_{n j}=b_{(n-1) j}+1$ and $b_{n i}=b_{(n-1) i}$ for $i \neq j$. The first three conditions are then clearly satisfied.

But let $n \in \mathbb{N} \cup\{0\}$ and define $j$ as above. We know $\sum_{i=1}^{m}\left(n b_{i}-b_{(n-1) i}\right)=1$, so $n b_{j}-b_{(n-1) j}>0$. Then $n b_{j}-b_{n j}=n b_{j}-b_{(n-1) j}-1>-1$. But, for $i \neq j$, $n b_{i}-b_{n i}=n b_{i}-b_{(n-1) i} \geq(n-1) b_{i}-b_{(n-1) i}>-1$ by induction, since, for all $i$, $0 b_{i}-b_{0 i}=0>-1$. Since $\sum_{i=1}^{m}\left(n b_{i}-b_{n i}\right)=0$ for all $n$, this implies the fourth condition.

Proof of Proposition [6. If $A$ is a generating set for $H$, then $\phi(A)$ is a generating set for $H_{a b}$, which we again identify with $\mathbb{Z}^{2}$. As in the discussion before Proposition 3 , let $B$ be the convex hull of $\pm \phi(A)$ and let $\|\cdot\|_{B}$ denote the norm with $B$ as its unit ball. Then Proposition 3 gives $C \in \mathbb{R}$ such that $\left|\|g\|_{\phi(A)}-\|g\|_{B}\right| \leq C$ for all $g \in \mathbb{Z}^{2}$.

Consider the polygon of maximal isoperimetric constant with respect to $\|\cdot\|_{B}$ given by Lemma 10. Denote this maximal isoperimetric constant by $M_{A}$. Choose $m$ sides of the polygon, taken in order. Scale the polygon to have perimeter 2 . It must have evenly many sides, so let $m$ be half its number of sides. Let $b_{1}, \ldots$, $b_{m} \in \mathbb{R}_{+}$be the lengths (in the norm $\|\cdot\|_{B}$ ) of the chosen $m$ sides; the lengths of the other $m$ sides are the same. Then $\sum_{i=1}^{m} b_{i}=1$, so choose $b_{n i}$ by Lemma 11 . Since each side of our original polygon was parallel to the projection under $\phi$ of an element of $A$, we get for each $n$ a word $w_{n}$ of length $2 n$ (thus satisfying the first condition)

$$
a_{1}^{b_{n 1}} \ldots a_{m}^{b_{n m}} a_{1}^{-b_{n 1}} \ldots a_{m}^{-b_{n m}}=[x, y]^{k_{n}},
$$

where the $a_{i} \in A \cup A^{-1}$ are independent of $n$ and $k_{n} \in \mathbb{Z}$. By the remark following Lemma 11 the $w_{n}$ satisfy the second condition as well. 
Each $k_{n}$ is (up to sign) the area of the polygon with pairs of opposite sides parallel to those of the original polygon but with lengths $b_{n i}$. This polygon will have perimeter $2 n$. But then

$$
\begin{aligned}
0 \leq 4 n^{2} M_{A}-\left|k_{n}\right| \leq \sum_{i=1}^{m}\left|b_{n i}-n b_{i}\right|\left(n+\sum_{j=1}^{m}\left|b_{n j}-n b_{j}\right|\right) & \\
<\sum_{i=1}^{m} m\left(n+m^{2}\right) & =m^{2}\left(n+m^{2}\right)
\end{aligned}
$$

But if we set $k_{\max }(A)=\max \left\{|k| \mid x^{i} y^{j}[x, y]^{k} \in A\right\}$ then $I_{A}(2 n) \leq 4(n+$ $C)^{2} M_{A}+2(n+C) k_{\max }(A)$. If $n=0$ then $I_{A}(2 n)=\left|k_{n}\right|=n^{2}=0$, while if $n>0$ then

$$
\begin{aligned}
0 \leq I_{A}(2 n)-\mid & k_{n} \mid \\
\leq m^{2}\left(n+m^{2}\right)+ & 8 n C M_{A}+4 C^{2} M_{A}+2 n k_{\max }(A) \\
\leq & \left(m^{4}+m^{2}+4 C^{2} M_{A}+8 C M_{A}+2 k_{\max }(A)\right) n
\end{aligned}
$$

and $m^{2}\left(n+m^{2}\right) \leq\left(m^{2}+m^{4}\right) n$. We may thus take $D=m^{4}+m^{2}+4 C^{2} M_{A}+$ $8 C M_{A}+2 k_{\max }(A), E=4 M_{A}$ and $F=m^{4}+m^{2}$.

This completes the proof of Proposition 1

\section{Proof of Proposition 2}

Proposition 2 will follow from

Proposition 12. Let $A$ be a generating set for $H$. Then there exists $C \in \mathbb{N}$ with the following property. Let $i, j, k_{1}, k_{2} \in \mathbb{Z}$ with either $i j / 2 \leq k_{1} \leq k_{2}$ or $i j / 2 \geq k_{1} \geq k_{2}$. Then

$$
\left\|x^{i} y^{j}[x, y]^{k_{1}}\right\|_{A} \leq\left\|x^{i} y^{j}[x, y]^{k_{2}}\right\|_{A}+C .
$$

This result in turn follows from another result, the statement of which requires the following

Definition 3. Let $A$ be a generating set for $H$. Let $i \phi(x)+j \phi(y) \in H_{a b}$ and $n$ be the minimal length with respect to $H$ of any element of $\phi^{-1}(i \phi(x)+j \phi(y))$. For $n^{\prime} \geq n$, let $k_{(i, j)}^{\max }\left(n^{\prime}\right)$ (respectively $\left.k_{(i, j)}^{\min }\left(n^{\prime}\right)\right)$ be the maximum (resp. minimum) $k$ such that there is a word of length $\leq n^{\prime}$ representing $x^{i} y^{j}[x, y]^{k}$.

Proposition 13. Let $A$ be a generating set for $H$. Then there are $D$ and $I \in \mathbb{N}$ with the following property. Let $i \phi(x)+j \phi(y) \in H_{a b}, n$ be the minimal length with respect to $H$ of any element of $\phi^{-1}(i \phi(x)+j \phi(y))$ and $n^{\prime} \geq n+I$. Then for every $k$ with $k_{(i, j)}^{\min }\left(n^{\prime}\right) \leq k \leq k_{(i, j)}^{\max }\left(n^{\prime}\right)$ there is $k^{\prime} \in \mathbb{Z}$ with $\left|k^{\prime}-k\right| \leq D$ such that $\left\|x^{i} y^{j}[x, y]^{k^{\prime}}\right\|_{A} \leq n^{\prime}$. Furthermore we have $k_{(i, j)}^{\min }\left(n^{\prime}\right) \leq i j / 2 \leq k_{(i, j)}^{\max }\left(n^{\prime}\right)$.

We postpone the proof.

Proof of Proposition 12, Let $D$ and $I \in \mathbb{N}$ be as in Proposition 13, Let $a_{1} \ldots a_{m}$ be a minimal-length word in the letters of $A$ and their inverses representing $x^{i} y^{j}[x, y]^{k_{2}}$. (Thus $m=\left\|x^{i} y^{j}[x, y]^{k_{2}}\right\|_{A}$.) It follows by definition that

$$
k_{(i, j)}^{\min }(m+I) \leq k_{(i, j)}^{\min }(m) \leq k_{2} \leq k_{(i, j)}^{\max }(m) \leq k_{(i, j)}^{\max }(m+I)
$$


and that $m+I \geq n+I$.

Assuming without loss of generality that $i j / 2 \leq k_{1} \leq k_{2}$, we see by the last sentence of Proposition 13 applied with $n^{\prime}=m+I$ that

$$
k_{(i, j)}^{\min }(m+I) \leq i j / 2 \leq k_{1} \leq k_{2} \leq k_{(i, j)}^{\max }(m+I) .
$$

and thus that there exists a $k^{\prime} \in \mathbb{Z}$ with $\left|k^{\prime}-k_{1}\right| \leq D$ such that

$$
\left\|x^{i} y^{j}[x, y]^{k^{\prime}}\right\|_{A} \leq m+I \text {. }
$$

Thus, by the triangle inequality,

$$
\left\|x^{i} y^{j}[x, y]^{k_{1}}\right\|_{A} \leq\left\|x^{i} y^{j}[x, y]^{k^{\prime}}\right\|_{A}+\left\|[x, y]^{D}\right\|_{A} \leq m+I+D\|[x, y]\|_{A} .
$$

We are done if we let $C=I+D\|[x, y]\|_{A}$.

Proof of Proposition [. For any $x^{i} y^{j}[x, y]^{k} \in H$, the sequence

$$
\left\{x^{i} y^{j}[x, y]^{k^{\prime}}\left|\left(k^{\prime}-k\right)(k-i j / 2)>0,\right| k^{\prime}-i j / 2|>| k-i j / 2 \mid\right\}
$$

has distance from the identity (with respect to any finite generating set) increasing without bound, since the metric with respect to any finite generating set is proper. However, consecutive elements are at $A$-distance $\|[x, y]\|_{A}$ from each other, and, by Proposition 12 applied to $k_{1}=k, k_{2}=k^{\prime}$, every element is at distance at least $\left\|x^{i} y^{j}[x, y]^{k}\right\|_{A}-C$ from the identity with respect to $A$. Thus we may take $r=C$.

We now proceed with the proof of Proposition 13, We will first need two lemmas.

Lemma 14. Let $A$ be a generating set for $H$. Then there exists $C \in \mathbb{N}$ with the following property. Let $n \in \mathbb{N}$ and $\left\{a_{1}, \ldots, a_{n}\right\}$ be a multiset of letters of $A$ and their inverses. Their projections to $H_{a b}$ have a well-defined sum, which we denote $i \phi(x)+j \phi(y)$. Then the average of the minimal and maximal $k$ such that some reordering $\sigma$ of the $a_{i}$ represents $x^{i} y^{j}[x, y]^{k}$ is within $C n$ of $i j / 2$.

Proof. Let $a_{t}=x^{b_{t}} y^{c_{t}}[x, y]^{k_{t}}$. Averaging over all $n$ ! possible choices for $\sigma$ gives an average $k$-value of

$$
\frac{\sum_{\sigma \in S_{n}} \sum_{1 \leq s<t \leq n} c_{\sigma(s)} b_{\sigma(t)}}{n !}+\sum_{t=1}^{n} k_{t}=\frac{n ! \sum_{1 \leq s \neq t \leq n} b_{s} c_{t}}{2 n !}+\sum_{t=1}^{n} k_{t},
$$

which differs (absolutely) from $\sum_{t=1}^{n} b_{t} \cdot \sum_{t=1}^{n} c_{t} / 2$ by

$$
\left|\sum_{t=1}^{n}\left(k_{t}-b_{t} c_{t}\right)\right| \leq n \max _{t=1}^{n}\left|k_{t}-b_{t} c_{t}\right| \leq n \max _{x^{b} y^{c}[x, y]^{k} \in A \cup A^{-1}}|k-b c| .
$$

The average of the minimal and maximal $k$-values equals the average $k$-value, since the distribution is symmetrically distributed about the average, as may be seen by considering what happens to $k$ when $\sigma$ is followed by the permutation which changes the order of every pair of elements. The result follows, letting $C$ be an integer greater than $\max _{x^{b} y^{c}[x, y]^{k} \in A \cup A^{-1}}|k-b c|$.

Lemma 15. Let $A$ be a generating set for $H$. Then there are $E \in \mathbb{R}_{>0}$ and $F \in \mathbb{N}$ with the following property. Let $i \phi(x)+j \phi(y) \in H_{a b}, n$ be the minimal length with respect to $H$ of any element of $\phi^{-1}(i \phi(x)+j \phi(y))$ and $d \geq 0$. Then there exists $\left\{a_{1}, \ldots, a_{n+d}\right\}$, a multiset of letters of $A$ and their inverses, such that $\sum_{t=i}^{n+d} \phi\left(a_{t}\right)=$ 
$(i, j)$ and the difference between the maximal and minimal possible exponent of $[x, y]$ obtainable by multiplying the $a_{t}$ in some order is $\geq f_{E, F}(n, d)=\max (E \max (n-$ $\left.F, 0) \max (d-F, 0), E d^{2}-F d\right)$. In particular, $k_{(i, j)}^{\max }(n+d)-k_{\min }(n+d) \geq f_{E, F}(n, d)$.

Proof. We show that the difference can be made to exceed each of the two expressions of which $f_{E, F}(n, d)$ is the maximum.

We will use $\|\cdot\|$ to denote the standard $L^{1}$ norm on $\mathbb{Z}^{2}$, which we identify with $H_{a b}$. It follows from Proposition 3 that there are $C$ and $D \in \mathbb{N}$ with the following property. Let $w$ be a word of length $n$ representing an element $h \in \phi^{-1}((i, j))$ and $v=\phi(h)$. Then there is $v^{\prime}$ with $\left\|v^{\prime}-v\right\| \leq C$ such that there is an element $h^{\prime} \in$ $\phi^{-1}\left(v^{\prime}\right)$ with $n-D \leq\left\|h^{\prime}\right\|_{A} \leq n+D$ and such that this length is attained by a word in $A \cup A^{-1}$ which is a product of letters projecting to at most two adjacent vertices of the unit ball obtained from $A$ by that proposition. Let $w^{\prime}$ be this word representing $h^{\prime}$. Since $\mathbb{Z}^{2}$ is not cyclic, the unit ball must have at least four vertices, hence at least two pairs of inverse vertices; we can thus choose such a pair (say $\pm p, p=\phi(a)$, $a \in A$ ) such that at most half the letters of $w^{\prime}$ project to $\pm p$. Write $w^{\prime}=a_{1} \ldots a_{m}$, where $n-D \leq m \leq n+D$ and the $a_{t} \in A \cup A^{-1}$. Let $k_{t}$ be such that $[x, y]^{k_{t}}=\left[a, a_{t}\right]$. The projections of the $a_{t}$, being at most two adjacent vertices of the unit ball, must all lie in one of the two closed half-planes bounded by $\langle p\rangle$. Thus $k_{t}$ is either nonnegative for every $t$ or nonpositive for every $t$. Assume without loss of generality that it is nonnegative. Furthermore, it is 0 for at most $m / 2$ values of $i$ by our choice of $p$. If $d>D$, then $a^{\lfloor(d-D) / 2\rfloor} w^{\prime} a^{-\lfloor(d-D) / 2\rfloor}$ represents $h^{\prime}[x, y]^{\lfloor(d-D) / 2\rfloor \sum_{t=1}^{m} k_{t}}$, while $a^{\lfloor(d-D) / 2\rfloor} w^{\prime} a^{\lfloor(d-D) / 2\rfloor}$ represents $h^{\prime}[x, y]^{-\lfloor(d-D) / 2\rfloor \sum_{t=1}^{m} k_{t}}$. Both these words have length at most $n+D+2\lfloor(d-D) / 2\rfloor \leq n+D+d-D=n+d$, and their exponents of $[x, y]$ differ by $\lfloor(d-D) / 2\rfloor \sum_{t=1}^{m} k_{t}$. If we let

$$
c_{\min }(A)=\min \left\{|k| \mid\left[a^{\prime}, a^{\prime \prime}\right]=[x, y]^{k}, a^{\prime}, a^{\prime \prime} \in A\right\},
$$

we get that the two words' exponents of $[x, y]$ differ by

$$
\begin{aligned}
& \left\lfloor\frac{d-D}{2}\right\rfloor \sum_{t=1}^{m} k_{t} \geq \frac{(d-D-1) c_{\min }(A) m}{2} \\
& \quad \geq \frac{c_{\min }(A)(d-D-1)(n-D)}{2} \geq \frac{c_{\min }(A)(d-D-1)(n-D-1)}{2} .
\end{aligned}
$$

Thus we are done with the first expression if we let $E=c_{\min }(A) / 2$ and $F=D+1$.

For the second expression, again let $w$, still of length $n$, represent $x^{i} y^{j}[x, y]^{k}$. Let $w_{x}$ and $w_{y}$ be minimal-length words in the letters of $A$ and their inverses representing $x$ and $y$, respectively. Then, if we let $l_{p}=2\left(l\left(w_{x}\right)+l\left(w_{y}\right)\right)$ we have

$$
w\left[w_{x}^{\left\lfloor\frac{d}{l_{p}}\right\rfloor}, w_{y}^{\left\lfloor\frac{d}{l_{p}}\right\rfloor}\right]
$$

and

$$
w\left[w_{y}^{\left\lfloor\frac{d}{l_{p}}\right\rfloor}, w_{x}^{\left\lfloor\frac{d}{l_{p}}\right\rfloor}\right]
$$

have length at most $n+d$ and represent $x^{i} y^{j}[x, y]^{k+\left\lfloor d / l_{p}\right\rfloor^{2}}$ and $x^{i} y^{j}[x, y]^{k-\left\lfloor d / l_{p}\right\rfloor^{2}}$, respectively. But

$$
2\left\lfloor\frac{d}{l_{p}}\right\rfloor^{2} \geq \frac{2 d^{2}}{l_{p}^{2}}-\frac{4 d}{l_{p}}
$$


so we are done with this expression if we let $E=2 / l_{p}^{2}$ and $F=4 / l_{p}$.

Thus, we may simply take the lesser of these two values of $E$ and an integer greater than or equal to these two values of $F$. The last sentence then follows trivially.

Proof of Proposition 13, For any word of length $n^{\prime}$ in the $A$ and their inverses, we can transform it to any permutation of itself by a succession of transpositions of adjacent letters. Each such transposition changes the exponent of $[x, y]$ by at most

$$
c_{\text {max }}(A)=\max \left\{|k| \mid\left[a_{1}, a_{2}\right]=[x, y]^{k}, a_{1}, a_{2} \in A \cup A^{-1}\right\} .
$$

Now consider a word $w_{\max }$ of length at most $n^{\prime}$ representing

$$
x^{i} y^{j}[x, y]^{k_{(i, j)}^{\max }\left(n^{\prime}\right)} \text {. }
$$

By Lemma 14, there is $C \in \mathbb{N}$ depending only on $A$ such that we can permute the letters of $w_{\max }$ so that it will represent $x^{i} y^{j}[x, y]^{k_{m}}$ with $\left(k_{(i, j)}^{\max }\left(n^{\prime}\right)+k_{m}\right) / 2 \leq$ $i j / 2+C n^{\prime}$. Thus $\left(k_{(i, j)}^{\max }\left(n^{\prime}\right)+k_{(i, j)}^{\min }\right) / 2 \leq i j / 2+C n^{\prime}$. Similarly, if $w_{\min }$ is taken to represent $x^{i} y^{j}[x, y]^{k_{(i, j)}^{m i n}\left(n^{\prime}\right)}$, then we can permute its letters so it will represent some $x^{i} y^{j}[x, y]^{k_{M}}$ with $\left(k_{(i, j)}^{\max }+k_{M}\right) / 2 \geq i j / 2+C n^{\prime}$, so $\left(k_{(i, j)}^{\max }\left(n^{\prime}\right)+k_{(i, j)}^{\min }\right) / 2 \geq i j / 2-C n^{\prime}$. It follows that $k_{(i, j)}^{\max }-k_{M} \leq 4 C n^{\prime}$ and $k_{m}-k_{(i, j)}^{\min } \leq 4 C n^{\prime}$. If (in the notation of Lemma 15) $f_{E, F}\left(n, n^{\prime}-n\right) \geq 8 C n^{\prime}$ for the appropriate $E \in \mathbb{R}_{>0}$ and $F \in \mathbb{N}$ (which depend only on $A$ ) then, by Lemma 15, $k_{(i, j)}^{\max }\left(n^{\prime}\right)-k_{(i, j)}^{\min } \geq 8 C n^{\prime}$, so the above inequalities imply $k_{M} \geq k_{m}$. Thus there will exist $k^{\prime} \in \mathbb{Z}$ with $\left|k^{\prime}-k\right| \leq c_{\max }(A) / 2$ such that some permutation of either $w_{\max }$ or $w_{\min }$ represents $x^{i} y^{j}[x, y]^{k^{\prime}}$, so we will be done.

It remains to note that we will have $E(n-F)\left(n^{\prime}-n-F\right) \geq 8 C n^{\prime}$ as soon as $n^{\prime} \geq E(n+F)(n-F) /[E(n-F)-8 C]$. But, if $n \geq 16 C / E+2 F$ we have

$$
\begin{aligned}
\frac{E(n+F)(n-F)}{E(n-F)-8 C} \leq(n+F)[ & \left.1+\frac{16 C}{E(n-F)}\right] \\
& \leq n+2 F+\frac{16 C}{E}\left(1+\frac{2 F}{n}\right) \leq n+2 F+\frac{32 C}{E},
\end{aligned}
$$

where we use repeatedly that if $0 \leq x \leq 1 / 2$ then $0 \leq 1 /(1-x) \leq 1+2 x$. If $n<16 C / E+2 F$, we will have $E\left(n^{\prime}-n\right)^{2}-F\left(n^{\prime}-n\right) \geq 8 C n^{\prime}$ as soon as $E\left(n^{\prime}-n\right)^{2}-F\left(n^{\prime}-n\right) \geq 8 C\left(n^{\prime}-n+16 C / E+2 F\right)$. This is a quadratic inequality in $n^{\prime}-n$, which will hold so long as $n^{\prime}-n \geq G$, say, where $G$ depends only on $A$. So we are done if we let $D$ be an integer $\geq c_{\max }(A) / 2$ and $I$ an integer $\geq \max (G, 2 F+32 C / E)$. The last sentence follows since if $f_{E, F}\left(n, n^{\prime}-n\right) \geq 8 C n^{\prime}$ then it is a fortiori $\geq 2 C n^{\prime}$, so since $\left(k_{(i, j)}^{\max }+k_{m}\right) / 2 \leq i j / 2+C n^{\prime}$ then $k_{m} \leq i j / 2$, and in the same way $k_{M} \geq i j / 2$.

This completes the proof of Proposition 2 .

\section{REFERENCES}

[1] M. Bestvina. Questions in geometric group theory. Available at http://www. math.utah.edu/ bestvina.

[2] O.V. Bogopol'skii. Infinite commensurable hyperbolic groups are bi-Lipschitz equivalent. Algebra and Logic, 36(3):155-63, 1997.

[3] S. Cleary and T.R. Riley. A finitely presented group with unbounded dead-end depth. Proceedings of the American Mathematical Society, 134(2):343-9, 2006. arXiv:math.GR/0406443. 
[4] S. Cleary and J. Taback. Dead end words in lamplighter groups and other wreath products. The Quarterly Journal of Mathematics, 56(2):165-78, 2005. arXiv:math.GR/0309344.

[5] J. Lehnert. Some remarks on depth of dead ends in groups. Preprint. arXiv:math.GR/0703636, 2007.

[6] T.R. Riley and A.D. Warshall. The unbounded dead-end depth property is not a group invariant. International Journal of Algebra and Computation, 16(5):969-83, 2006. arXiv: math.GR/0504121.

[7] A.D. Warshall. Deep pockets in lattices and other groups. Preprint. arXiv:math.GR/0611575, 2007.

Yale University, Department of Mathematics, P.O. Box 208283, New Haven, CT 06520-8283, USA

E-mail address: andrew.warshall@yale.edu 